\title{
Concentrado artesanal: una alternativa para la alimentación de aves (Gallus gallus domesticus) en razas de doble propósito
}

\author{
Felipe Santiago Salvador Bonilla \\ Ingeniero Agrónomo \\ Investigador \\ Universidad de Oriente, El Salvador \\ fsalvador@univo.edu.sv
}

Recepción: 27/04/2017

Aceptación: 08/07/2017

\section{Resumen}

El ambiente agropecuario en comunidades rurales en la Zona Oriental de El Salvador adopta un modelo basado en la producción de autoconsumo, implicando la seguridad alimentaria y nutricional del núcleo familiar. En esa dinámica, la Universidad de Oriente realizó un proyecto de investigación en el área de agropecuaria y medio ambiente que tuvo como objetivo evaluar una fórmula de concentrado artesanal, que mejora la producción de huevo en razas de gallinas de doble propósito.

Se preparó un alimento utilizando harina de maíz, sorgo, melaza, minerales y vitaminas, empleando un producto farmacéutico (Nutravit), complementado con la adición de la hoja de Madre cacao (Gliceridia sepium) en forma de harina en diferentes porcentajes. Se suministró este alimento a dos razas de gallina de doble propósito conocidas como Plymouth Rock Barrada y Rodhe Island Red, en las cuales se midieron variables de repuesta como peso de la gallina, postura; peso y masa del huevo, y su relación costo/beneficio, durante diez semanas; utilizando un diseño completamente al azar. Se determinó que la dosis que contenía un 5\% de harina preparada con hojas de Madre cacao tiene buena aceptabilidad de consumo en las gallinas. También se observó que influyó en que la masa de huevo alcanzara un mayor porcentaje y una buena pigmentación, mientras que el análisis estadístico determinó para las otras variables medidas diferencias no significativas.

Palabras clave: avicultura de traspatio, seguridad alimentaria, Madre cacao, Plymouth Rock Barrada, Rodhe Island Red.

\begin{abstract}
The farming environment in rural communities on the Eastern part of El Salvador adopts a model based on the self-consumption production involving food and nutritional security for the nuclear family. In that dynamic, the Universidad de Oriente carried out a research project in the farming area and the environment that had as objective to evaluate a formula of artisan concentrate that improves the egg production in different hen varieties of double purpose.
\end{abstract}

A kind of food was prepared using corn flour, sorghum, molasses, minerals and vitamins implementing a pharmaceutical product (Nutravit) complementing it with the Madre cacao leave (Gliceridia sepium) in the form of flour in different portions. This food was provided to two hen's varieties of dual purpose best known by Plymouth Rock Barrada and Rodhe Island Red in which were measured variable answers as the hen weight, posture, egg mass and weight and also its cost/benefit relation, during ten weeks using a random design. It was determined that the doses that contained $5 \%$ of processed flour with Madre cacao leaves have good ingestion acceptability on the hens. Besides, it was observed an influence inasmuch as the egg mass reached a higher proportion and a good pigmentation while the statistics analysis determined for the rest of the variables different non-significant consequences.

Key words: backyard poultry farming, food security, Madre cacao, Plymounth Rock Barrada, Rodhe Island Red. 


\section{Introducción}

La avicultura de traspatio constituye un recurso importante en el sistema de producción de la familia salvadoreña, está basada en la crianza de gallinas 'criollas'; y vinculada de manera directa con aspectos culturales, convirtiéndola en la base de la seguridad alimentaria local. Según el Instituto de Nutrición de Centro América y Panamá (INCAP), el consumo de huevo a nivel nacional es de $23 \mathrm{~g} / \mathrm{dia}$ rios, veinticuatro en zona urbana y veintiún en la zona rural. El consumo de carne de aves es de $20 \mathrm{~g} /$ diarios, veinticinco en la zona urbana y once en la zona rural. Estos datos evidencian que la ingesta, tanto de carne como de huevo en la zona rural proviene de las aves de traspatio (Menchú y Méndez, 2011).

Es conocido que la producción de estas aves se realiza de forma rústica, sin ningún control en el mejoramiento genético, salud y alimentación. Como resultado de esta dinámica productiva, instituciones como la Organización de las Naciones Unidas para la Alimentación y la Agricultura (FAO) y el Centro Nacional de Tecnología Agropecuaria y Forestal "Enrique Álvarez Córdova” (CENTA), impulsan programas que permitan mejorar los conocimientos de la población para manejar estos recursos. Entre éstos programas se encuentran el Proyecto Especial de Seguridad Alimentaria (PESA) y el Plan de Agricultura Familiar (PAF), introduciendo en algunos municipios del país razas de gallinas de doble propósito (carne y huevo), proponiendo el encierro para el manejo de las aves.

Esta iniciativa ha presentado dificultades para las familias, ya que las aves se trasponen (interrumpen su ciclo de postura) y bajan de peso. El conocimiento empírico señala que estas situaciones podrían estar asociadas a deficiencias en los requerimientos nutricionales que se les aportan. Entonces, surge la necesidad de buscar alternativas de solución que mejoren la alimentación de este tipo de aves cuando se mantienen en encierro.

La etapa experimental consistió en probar dosis de Madre cacao como fuente de proteína que mejore la producción de huevo y carne. La fórmula del concentrado artesanal estuvo fabricado a base de harina de maíz, sorgo, hoja de Madre cacao, energía y minerales. Fue probado en dos razas de gallinas de doble propósito: Rhode Island Red y Plymouth Rock Barrada.

\section{Materiales y Métodos}

El proceso de investigación, se inició en enero y finalizó en diciembre del año 2015. La fase experimental se realizó en el Campo de la Universidad de Oriente ubicado en el Cantón Anchico, Jurisdicción del departamento de San Miguel, El Salvador cuyas coordenadas son las siguientes: Longitud Oeste 88.76' ', y Latitud Norte de $13.26^{\prime \prime}$, a una altura de 110 
m.s.n.m, a $16 \mathrm{kms}$ de la ciudad de San Miguel, rumbo sureste, El Salvador.

Los componentes que formaron parte de las raciones alimenticias fueron: harina de maíz, sorgo, Madre cacao (de hojas deshidratadas); se le incorporó un compuesto que contiene una mezcla de vitaminas, electrolitos y aminoácidos solubles en agua (Nutravit). Además, se les adicionó melaza que es una sustancia espesa, dulce y de color oscuro, que queda como residuo de la cristalización del azúcar de caña.

Las actividades previas al ensayo fueron: desinfección y construcción de divisiones en la galera para unidades experimentales, colocación de camada, elaboración de concentrado artesanal y estabilización de las gallinas a la dieta alimenticia.

Se utilizó un diseño completamente al azar, con cuatro tratamientos descritos de la siguiente manera: T0: Harina de maíz 49\% + harina de sorgo $49 \%$ + Nutravit y melaza $2 \%$. T1: Harina de maíz 46.5\% + harina de sorgo $46.5 \%+$ harina Madre cacao 5\% + Nutravit y melaza 2\%.T2: Harina de maíz 45.5\% + harina de sorgo $45.5 \%$ + harina Madre cacao $7 \%$ + Nutravit y melaza 2\%.T3: Harina de maíz $44 \%$ + harina de sorgo $44 \%$ + harina madrecacao $10 \%+$ Nutravit y melaza $2 \%$. Cada tratamiento se replicó cuatro veces.
El contenido nutricional por cada dieta alimenticia estuvo determinado para T0: proteína $13.4 \%$, fibra $2.5 \%$, grasa $2.7 \%$, humedad $12.3 \%$. T1: proteína $14.2 \%$, fibra $2.7 \%$ grasa $3.0 \%$, humedad $12.5 \%$.T2: proteína $15.6 \%$, fibra $3.1 \%$ grasa $2.8 \%$, humedad $12.6 \%$. T3: proteína $16.3 \%$, fibra $3.4 \%$ grasa $3.1 \%$, humedad $13.1 \%$.

Las gallinas se alojaron en una galera de $11 \times 5 \mathrm{~m}$, dividida en dieciséis compartimientos con una medida $0.96 \times 2.5 \mathrm{~m}$; se contó con espacio central dentro de la galera de $11 \times 1.85 \mathrm{~m}$ para labores de manejo. Cada unidad experimental estuvo conformada por cinco gallinas, haciendo un total de 80 aves en estudio, de las cuales 20 fueron de la raza Plymouth Rock Barrada y 60 de Rhode Island Red, con una edad promedio de 5.5 meses. Se midieron las variables peso de la gallina, postura, peso y masa del huevo, pigmentación de la yema y relación costo/beneficio. A las observaciones se les aplicó el análisis de varianza (ANVA), haciendo uso del programa estadístico Excel (2010). A los efectos significativos de las variables cuantitativas en estudio se les realizó la prueba de rango múltiple DUNCAN. El ensayo tuvo una duración de diez semanas.

El manejo del ensayo durante el proceso de experimentación consistió en tener las aves en encierro; para ello se colocaron bebederos y comederos, ahí se les proporcionaba diariamente el alimentos las mañanas, al mismo tiempo que se les colocaba agua limpia. 
El concentrado suministrado a las aves estuvo constituido por harina de maíz y sorgo, harina de hoja de Madre cacao a dosis predeterminadas por la teoría. Además, se le adiciona melaza, sal y minerales; a excepción del tratamiento testigo, no se le adicionaba harina de hoja de madre cacao. Es importante señalar que en cuanto a los cuidados de salud de las aves se siguió un plan profiláctico con el objetivo de mantener las gallinas en condiciones óptimas, de tal forma que pudieran responder bien a los suplementos alimenticios.

\section{Resultados y Discusión}

\section{a. Peso de gallina $(\mathrm{Kg})$}

Esta característica se midió cada quince días a partir del inicio del ensayo, utilizando una balanza tipo reloj bien calibrada para obtener precisión en la medición de los datos con el objetivo de tener un parámetro del peso de las aves. Se tomó en cuenta que en este tipo de aves de doble propósito, este es un indicador de importancia para las familias productoras, (ver Figura 1).

Los resultados fueron sometidos al estadístico Análisis de Varianza, demostrándose que no existen diferencias entre los tratamientos a un nivel de significancia del $5 \%$, puesto que se encontró un P-value > 0.05 / 0.313 .

Es importante destacar que el peso es un factor determinante en las gallinas de doble propósito, debido a que son consideradas productoras de carne. Al observar la Figura 1 se puede apreciar que al inicio del ensayo, la raza Plymouth Rock Barrada presentaba un buen promedio de peso de $1.8 \mathrm{Kg}$; mientras

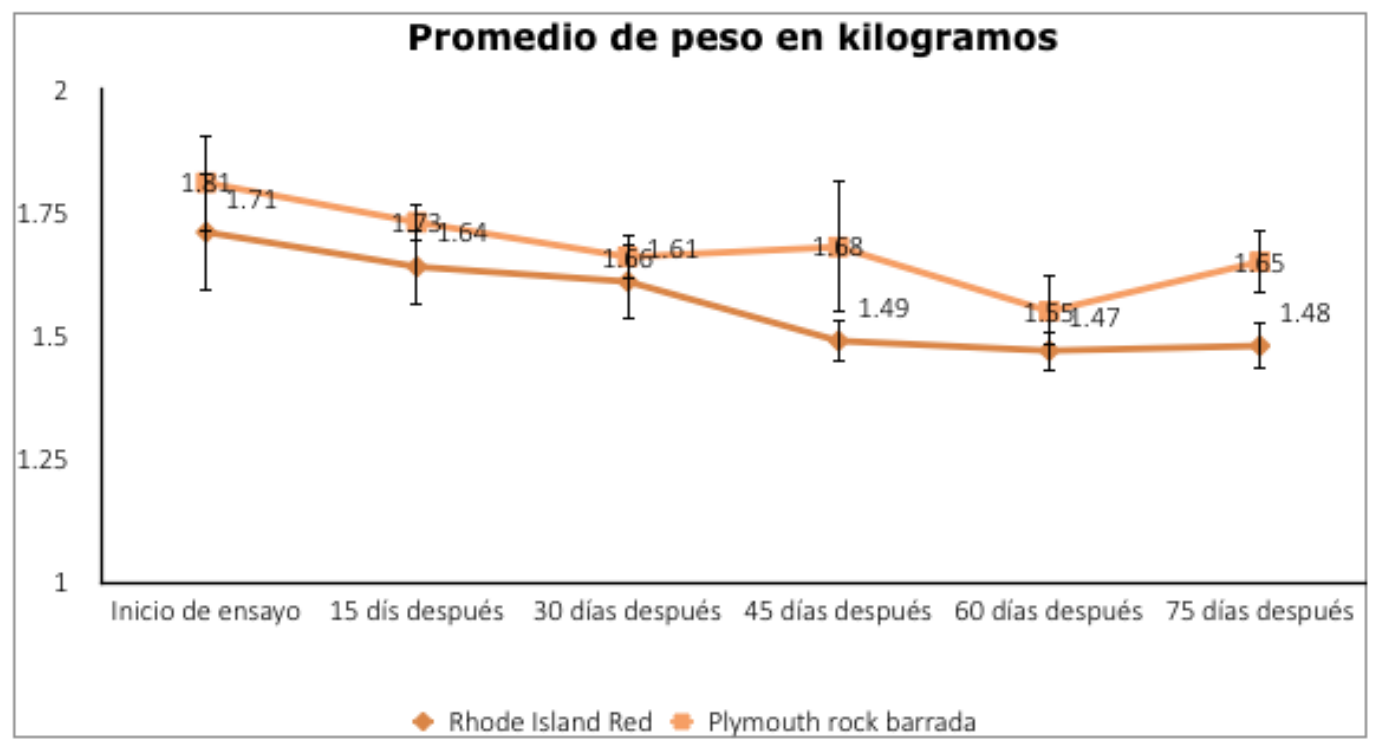

Figura 1. Evaluación del peso de la gallina durante diez semanas. 
que la Rhode Island Red 1.7Kg. A medida que transcurría el ensayo se ve un descenso en el peso, atribuible a que las gallinas iniciaron su ciclo de postura, y se observó una recuperación en la última semana, coincidiendo con la baja del índice de postura.

Con respecto a la influencia de los porcentajes de harina de Madre cacao incluidos en los tratamientos, se puede afirmar que ésta no influye en el peso, ya que estadísticamente resultaron ser iguales. Cabe señalar que el descenso en el peso se hace evidente cuando aumenta su índice de postura y encluecan ${ }^{1}$.

Con el objetivo de tener un parámetro en cuanto al peso que permita identificar los tipos de aves que se deben producir en las comunidades rurales de la zona, la Figura 2 presenta una comparación entre gallinas con- sideradas como criollas, ponedoras y las de doble propósito.

Es evidente que las gallinas de doble propósito presentan un buen peso, incluso con un buen manejo alimenticio, se podrían encontrar valores por sobre los obtenidos en el ensayo. Cabe señalar que el efecto de la adición de la harina de Madre cacao influyó en la cantidad diaria de consumo de alimento de las aves.

En la evaluación de dos dietas en gallinas criollas y mejoradas, en semi confinamiento sobre la postura en el municipio de Yuscarán, Honduras, se encontró que las aves criollas alcanzaron un peso de $1.55 \mathrm{~kg}$, mientras que las mejoradas $2.01 \mathrm{~kg}$.

Se puede decir que la genética y la alimentación inciden en el peso de las aves, conocien-

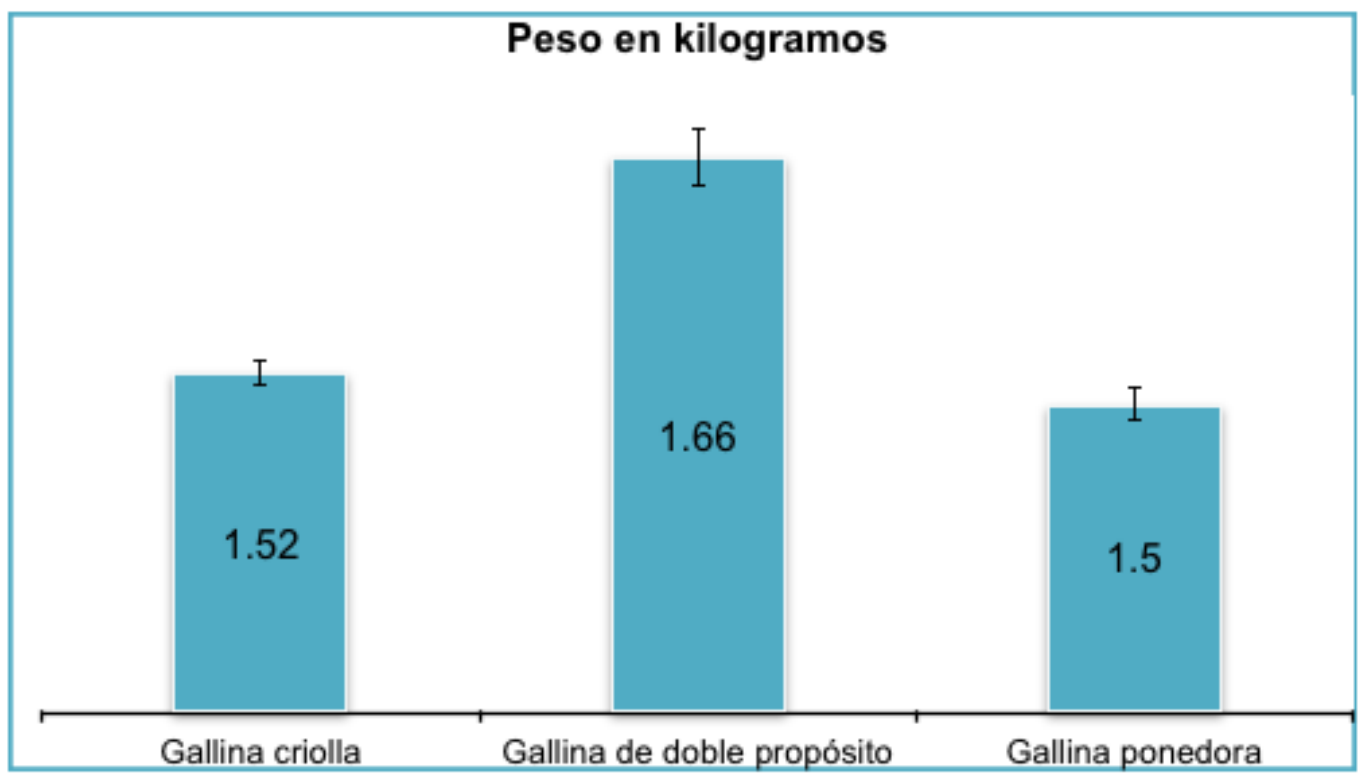

Figura 2. Evaluación del peso en tres tipos de gallinas

1. Estado fisiológico normal de las hembras de las aves en que dejan de poner huevos y se concentran en su incubación una vez que han completado la nidada. 


\section{Promedio de número de huevos}
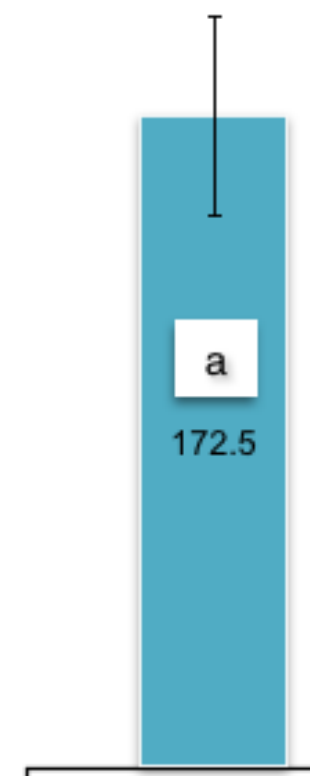

TO T1 $\mathrm{T} 2$

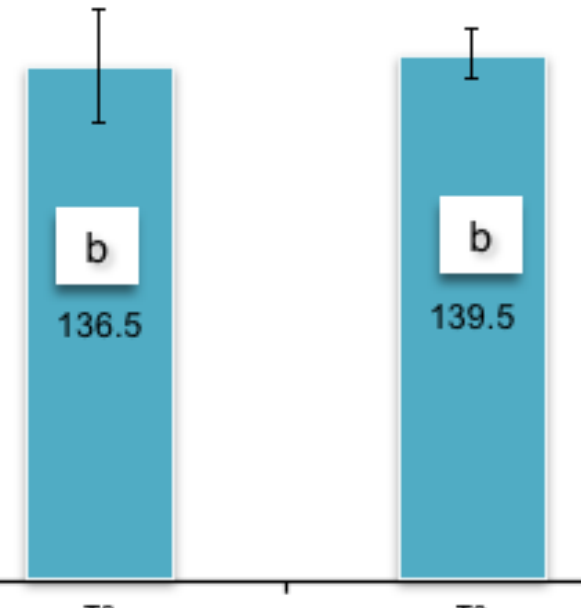

$\mathrm{T} 3$

Figura 3. Evaluación de postura de huevos por tratamiento

do que las gallinas criollas, por su condición genética, presentan características de bajo peso, siendo lo contrario en las mejoradas. Ésta es una condición deseable en este tipo de ave, constituyendo una mejora significativa en la productividad de las familias en la zona rural (Aguilar, 2001).

\section{b. Postura del huevo}

Se sabe que el número de huevos puesto por una gallina determina su potencial productivo. En el ensayo de esta variable se tomaron datos cada semana, y al final se promediaron los resultados por tratamientos (Figura 3).

Los datos encontrados fueron sometidos al Análisis de Varianza (ANVA), y resultó que existen diferencias significativas entre los tra- tamientos a un nivel de significancia del 5\%, puesto que el $\mathrm{P}$-value $<0.05 / 0.028$.

Al encontrar desigualdades significativas entre los tratamientos fue necesario aplicar la prueba de Duncan, determinando las siguientes diferencias por tratamiento: el testigo que no tenía harina de Madre cacao resultó ser estadísticamente no diferente con el tratamiento que poseía un 5\%; mientras que el tratamiento que tenía $7.0 \%$ y 10\% presentó un comportamiento igual estadísticamente. Otro aspecto cuantitativo importante fue el comportamiento semanal de postura por razas, los que son mostrados en la Figura 4. 
Un factor de influencia en la producción de huevo en las razas de doble propósito es la alimentación. Como lo plantea Pontes Pontes y Castello Lloobet (1995), a la gallina deben suministrársele todos los nutrientes necesarios para que responda de manera satisfactoria a los objetivos de producción. Por comparación, se determinó que los tratamientos más efectivos en la puesta de huevos fueron el testigo y el uno: éste contenía un 5\% de harina de hoja de Madre cacao, como se observa en la Figura 3. Estos resultados son comparables a los datos teóricos conocidos de una raza de doble propósito en la que su postura anual ronda los 150 a 200 huevos al año. Cabe apuntar que los tratamientos a los que se les incrementó el porcentaje de harina de hoja de madre cacao no aumentaron el índice de postura. Esto se explica considerando la menor aceptación en el consumo, que obtuvo un promedio de 2.5 onzas diarias, cuando se tiene estipulado que una gallina en esa fase de postura consume 3.75 onzas diarias.

Estos resultados superan los encontrados en otra investigación realizada en producción de huevo de gallinas Rhode Island Rojas bajo un sistema alternativo de traspatio, con una producción promedio de 2.3 huevo/ave/semana. (Salas y Rodríguez, 2009).

Los hallazgos significativos del estudio en la postura de huevo también estuvieron influenciados por la raza. Al observar la Figura 4, donde se hace una comparación entre las dos razas mencionadas, se puede apreciar que la raza Rhode Island Red presentó mejor tendencia de postura en número de huevos, efec-

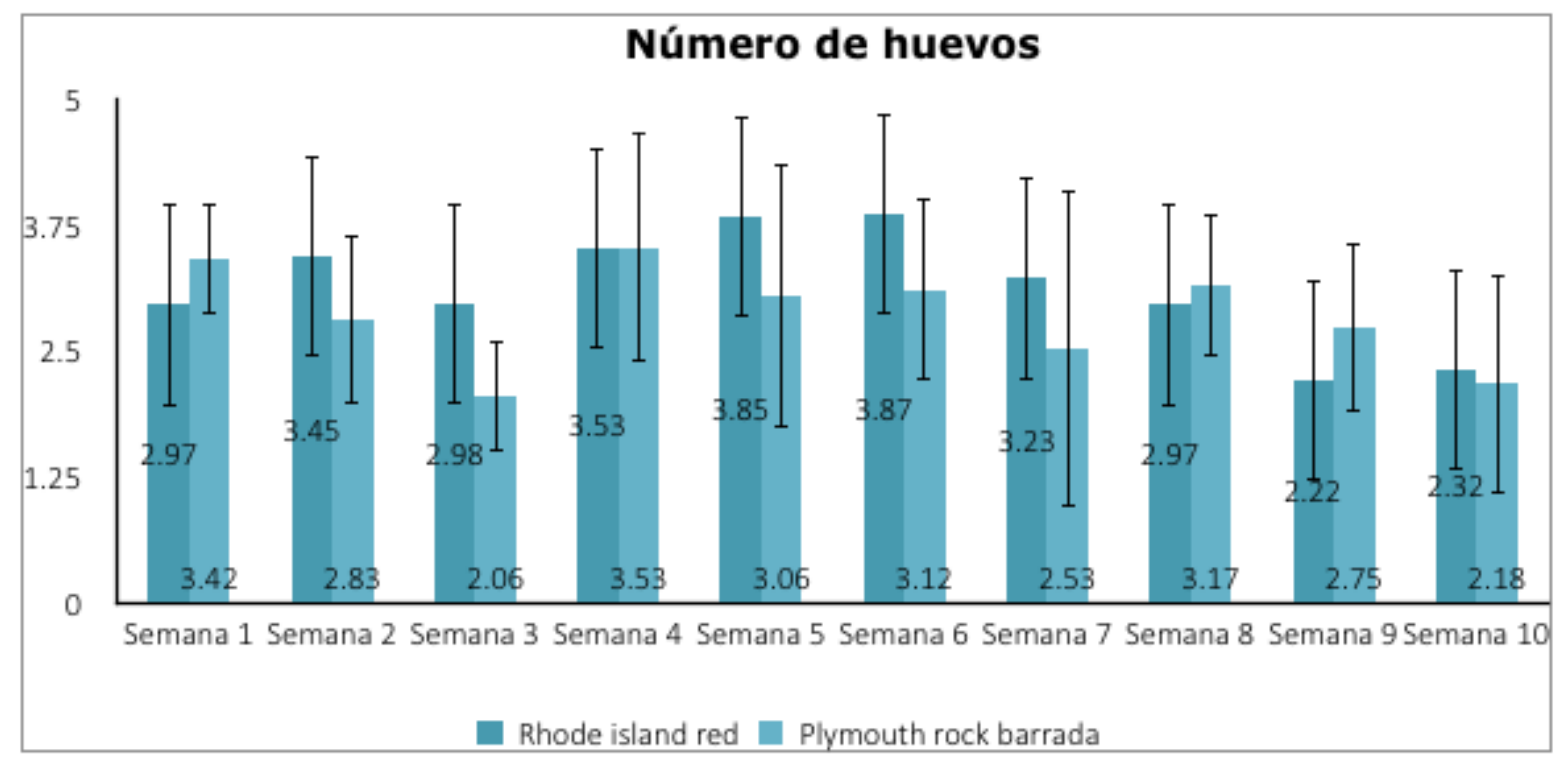

Figura 4. Evaluación del número de huevos por razas 
to que podría considerarse a la adaptabilidad de estas razas al tipo de alimentación que se le suministró.

\section{c. Peso del huevo (g)}

El peso constituye una variable importante, considerando que determina el tamaño. En el ensayo se hizo una evaluación semanal del peso en gramos. Al someter los resultados al análisis estadístico se encontró que solo en la primera toma de datos existen diferencias significativas entre los tratamientos, obteniendo un P-value < 0.05/0.0019. Se aplicó la prueba de Duncan, determinándose que los mejores resultados fueron para el tratamiento 2, al que se le había adicionado el 7.0\% de harina de hoja de Madre cacao. Para comprender mejor los resultados se presentan las Figuras 5,6 y $7:$

Es evidente que en las gallinas ponedoras el peso es mayor, considerando que su

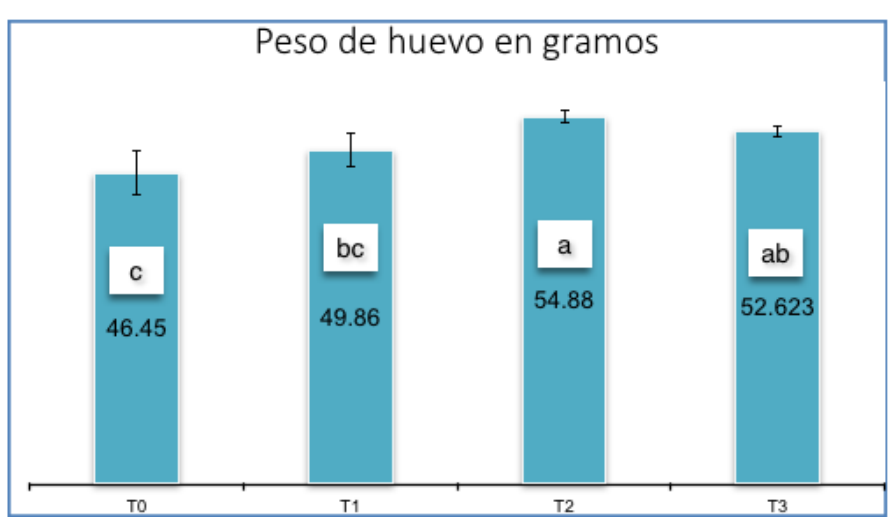

Figura 5. Evaluación de peso promedio de huevo/gramos por tratamiento. razas en evaluación osciló entre los 50 y 58 gramos, condición que supera los valores encontrados en una evaluación de aves criollas y líneas mejoradas con dos tipos de alimentación sobre la postura, realizada en la Escuela Agrícola Panamericana Zamorano, Honduras. En el peso de los huevos, la media en las líneas mejoradas fue de 47.10 gramos y en la criolla de 42.78 gramos. (Marañón B., 2001).

Es importante señalar que el peso del huevo fue mayor en aquellos tratamientos que contenían harina de hoja de Madre cacao. Otro aspecto relevante es que supera el peso de huevos de gallinas criollas. Para establecer esta comparación se tomó una muestra de gallinas de traspatios cercanos a donde se realizó el ensayo. Además, en investigaciones hechas en municipios de México, en este mismo tipo de aves criollas, se encontraron resultados entre 47.70 y 57.40 gramos. (Juárez-Caratagenética y la dieta alimenticia suministrada son diseñadas para ese fin. Sin embargo, es importante analizar los resultados obtenidos en el ensayo, ya que al observar las mediciones de los datos a partir de la segunda semana, el peso de las dos chea, Gutiérrez-Vázquez, Segura-Correa y Santos-Ricalde, 2010).

Con base a los resultados es favorable el mejoramiento del peso del huevo, utilizando dietas alternativas en la alimentación de las aves 


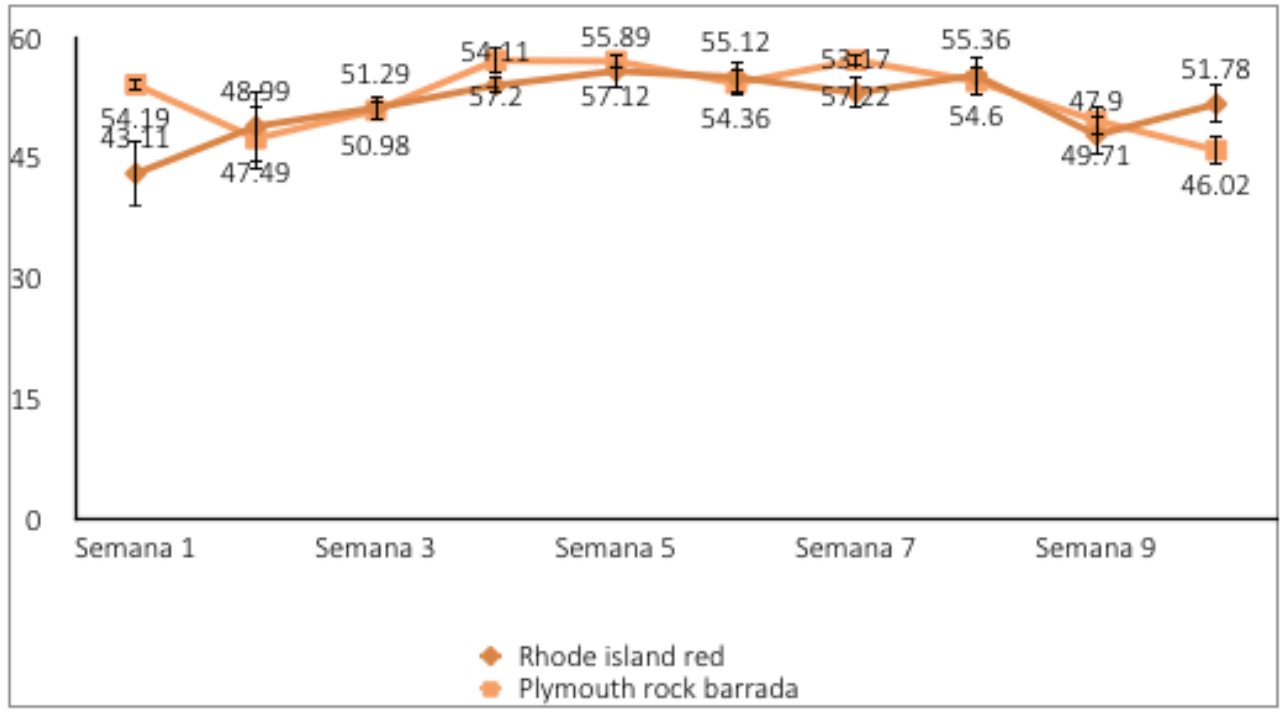

Figura 6. Evaluación peso de huevo/gramos por razas

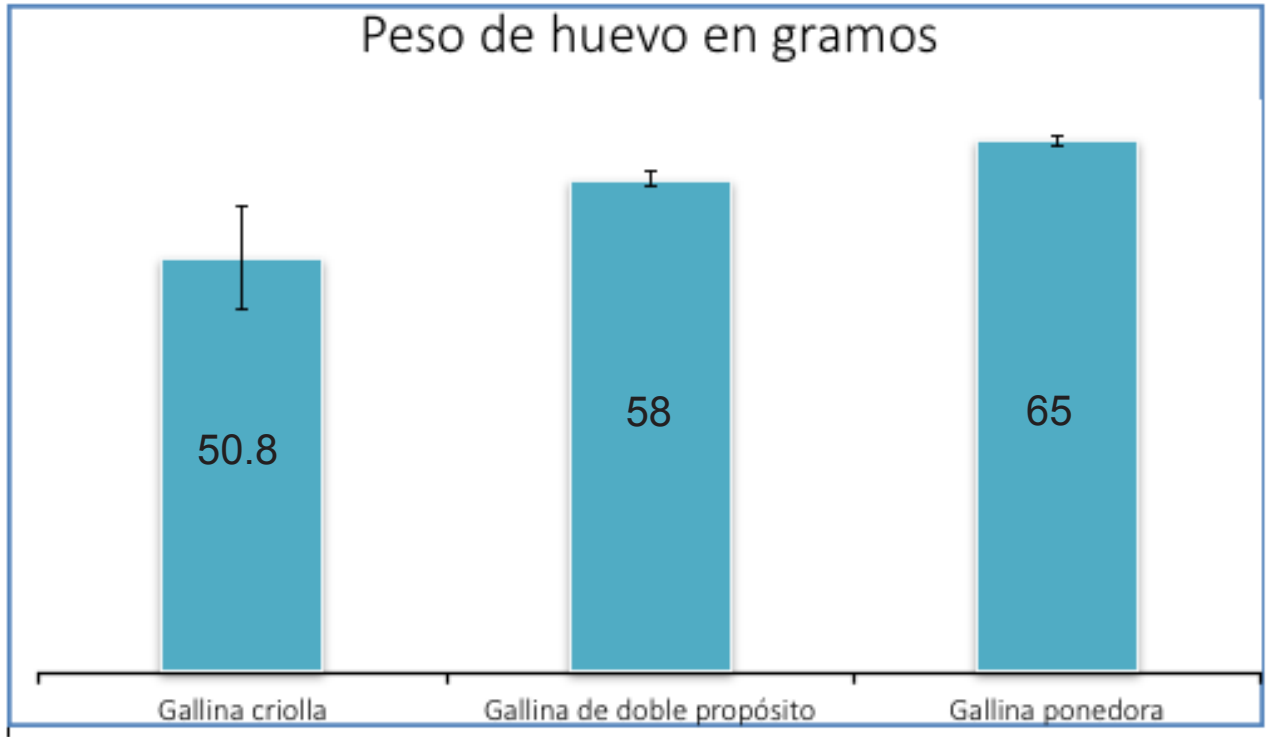

Figura 7. Comparación peso de huevo/gramos en tres tipos de gallinas

de doble propósito condición que permite una producción sustentable para la productividad de las familias que tengan la intención de producir este tipo de aves como forma de autoconsumo y comercialización con el objetivo de mejorar su economía familiar.

\section{d. Masa de huevo (\%)}

La masa del huevo está constituida por la parte comestible, conocida como el albumen o clara y la yema, considerando la necesidad de terminar en porcentajes de la cantidad de masa de huevo que tanto podrá influir la alimentación o la raza de la misma ave. 


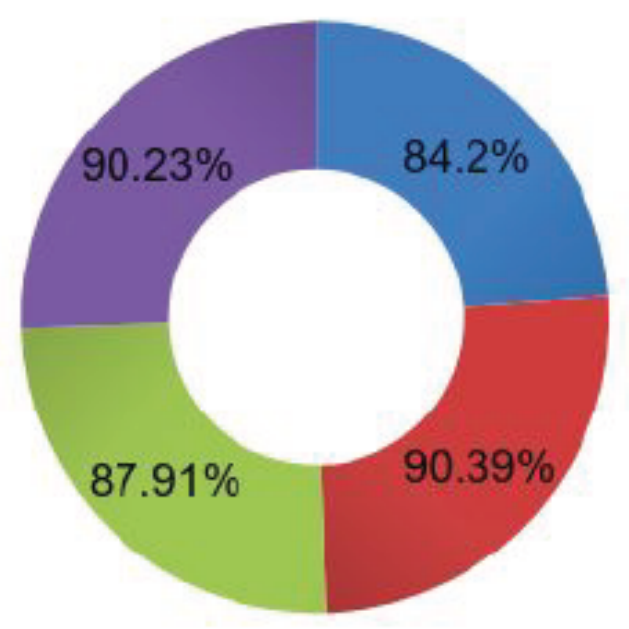

T0: Harina de maiz $49 \%$ + harina de sorgo $49 \%$ + minerales, vitaminas y energia $2 \%$.

T1: Harina de maiz $46.5 \%+$ harina de sorgo $46.5 \%$ + harina madrecacao $5 \%$ + minerales, vitaminas y energia $2 \%$. T2: Harina de maiz $45.5 \%$ + harina de sorgo $45.5 \%$ + harina madrecacao $7 \%$ + minerales, vitaminas y energia $2 \%$. T3: Harina de maz $44 \%$ + harina de sorgo $44 \%$ + harina madrecacao $10 \%$ + minerales, vitaminas y energia $2 \%$.

Figura 8. Evaluación del porcentaje de la masa del huevo.

Esta variable se midió tomando una muestra por tratamiento, cuyos datos se presentan en la Figura 8. Los resultados aparecen en porcentajes, tomándose el 100\% del peso de huevo para cada tratamiento:

La interpretación de la figura permite afirmar que el tratamiento uno y tres presentaron el mayor porcentaje de masa de huevo, mientras que el testigo obtuvo el menor, lo que lleva a considerar que la harina preparada con hojas de Madre cacao influye en el peso del huevo. También se puede denotar que el tratamiento uno superó al dos, aunque el primero tenía una menor dosis de esta harina, razón que podría atribuirse a que en el tratamiento dos quedó la mayoría de unidades experimentales conformadas por la raza Plymouth Rock Barrada; ésta presentó menor aceptación al consumo de concentrado que tenía adicionado porcentajes de esta sustancia. Asimismo, la masa del huevo es un factor que incide para determinar su calidad.

En otra investigación en suplementación de dietas de gallinas de postura comercial con aceites de pescado de diferentes grados de refinación, se encontraron efectos productivos en las aves y en la calidad organoléptica de los huevos. En los porcentajes de la masa del huevo se encontraron valores que oscilaron entre 64 y 76.14\%. Esto manifiesta que los valores encontrados en el ensayo son superiores a los del ensayo citado, lo que podría derivarse del efecto de la harina de Madre cacao y las razas evaluadas (Cornejo, Hidalgo, Araya, y Pokniak, 2008).

\section{e. Pigmentación en la yema de huevo}

El color de la yema de huevo juega un papel importante para su aceptación en el mercado. Se sabe que en nuestro medio y en la mayoría 
de localidades del mundo, el color amarillo-dorado es el preferido. Según Sandoval, Romero y Eguaras, J. L. (1966), la cantidad de carotenoides es la responsable de esa coloración. Ellos se dividen en los de coloraciones amarillas como luteína, zeaxantina, apo-ester, y la cantaxantina, responsable de la coloración roja.

Los resultados de pigmentación encontrados en el ensayo por efecto de los tratamientos, se presentan haciendo una comparación con una imagen de los rangos del ordenamiento de los carotenoides y su intensidad en su coloración, (ver figura 10).

Los resultados de la pigmentación de la yema de huevo en el ensayo por efecto de adicción de harina de Madre cacao inciden en la co- loración. Si se compara con la imagen en la Figura 9, se podría decir que los carotenoides presentes en el tratamiento testigo (T:0) son bajos, observándose una coloración amarillo pálido, mientras que aquellos en los cuales se suministró harina de Madre cacao presentaron una coloración amarilla más intensa.

Según la escala de valores de los carotenoides se puede afirmar que la harina de hoja de Madre cacao aporta los carotenoides luteína, apo-ester y zeaxantina, de forma natural, mejorando así el aporte de vitaminas. Los valores de la cantaxantina, carotenoides responsables de la coloración roja de la yema, se presentaron en baja intensidad. Se comparó la coloración de la yema de huevo del ensayo con aves alimentadas con concentrado comercial,

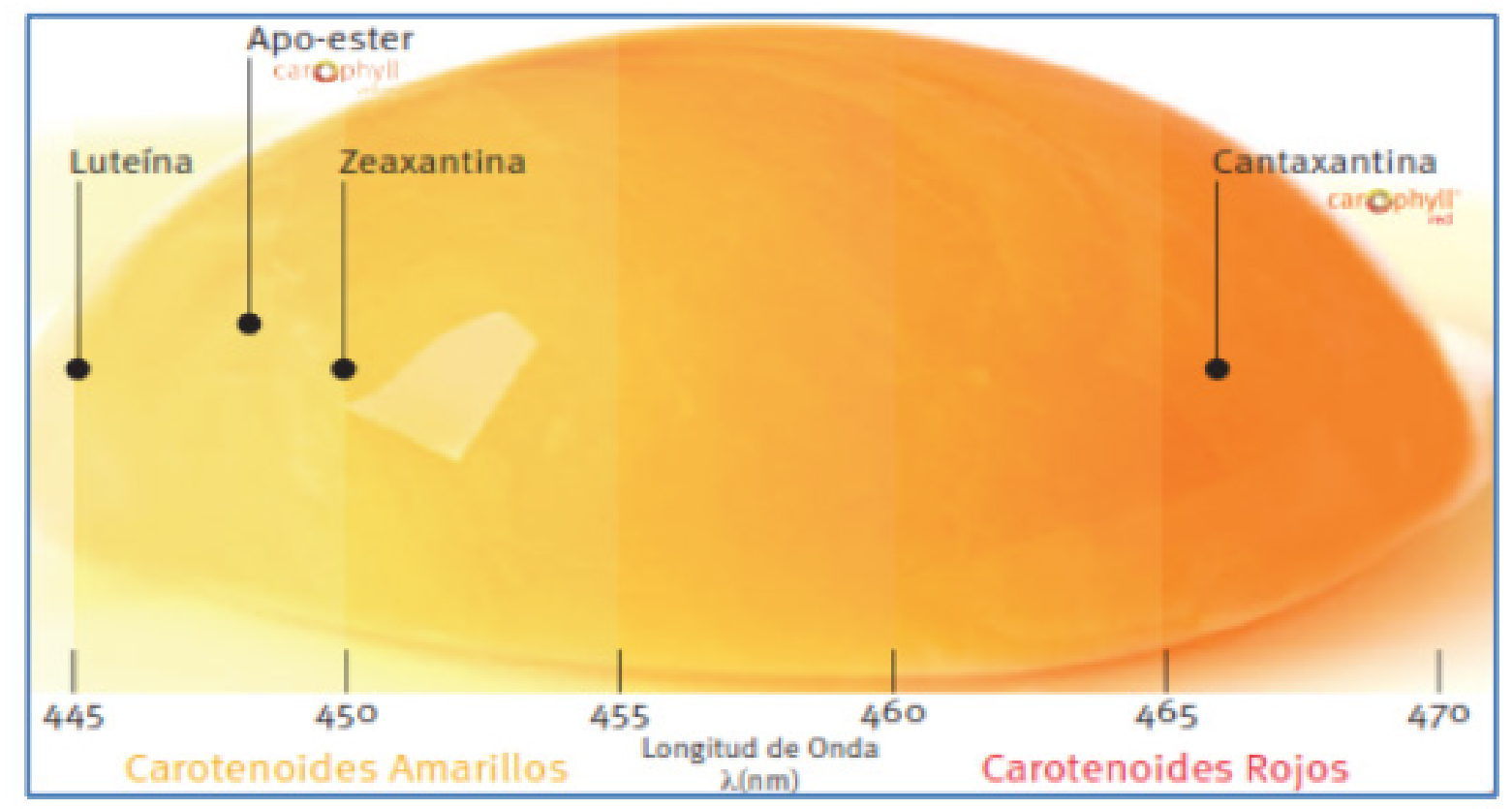

Figura 9. Longitudes de hondas para varios carotenoides utilizados para la pigmentación de la yema de huevo.

Fuente: Tomado de DSM, CARIOPHILL 


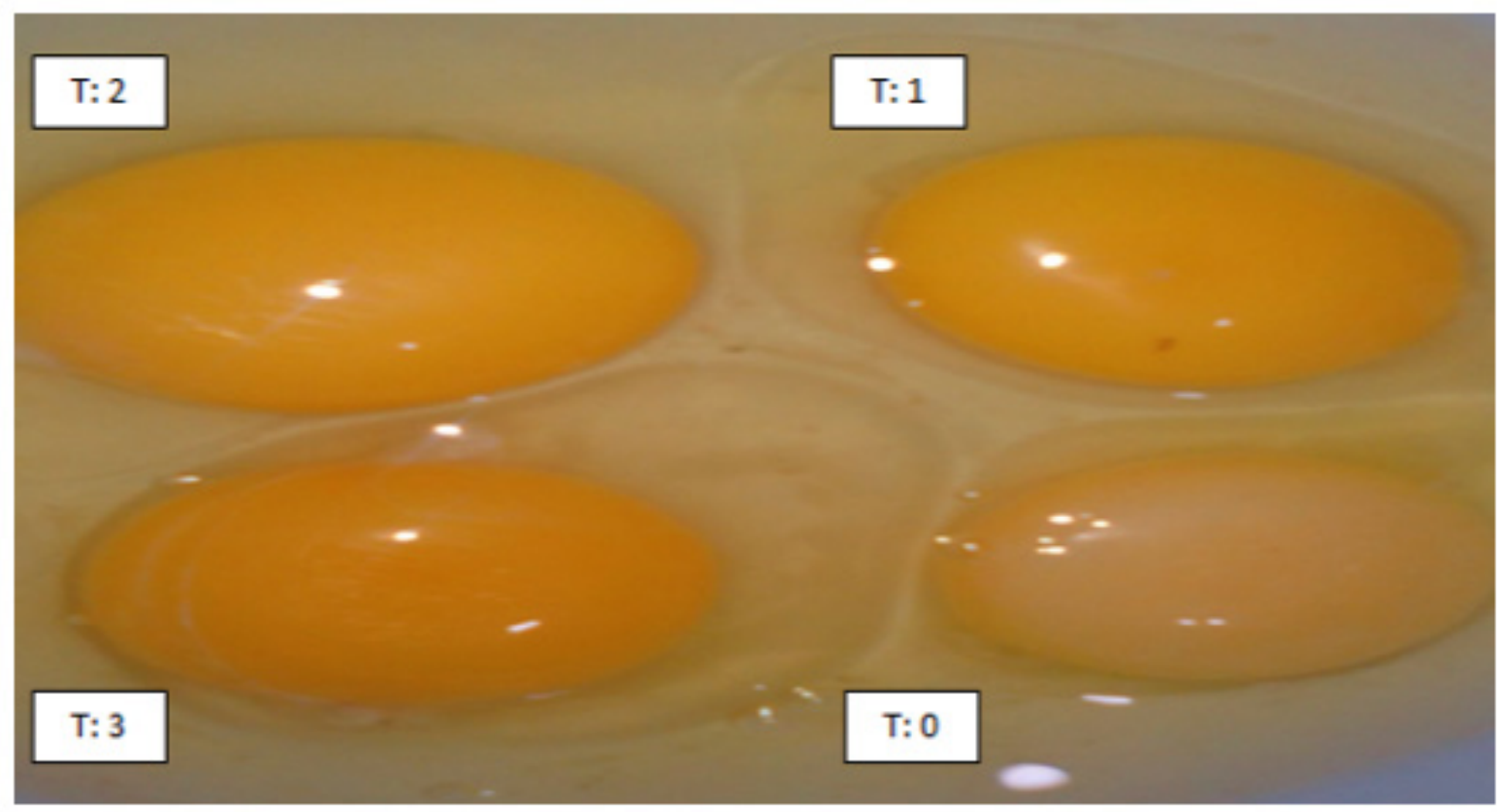

Figura 10. Evaluación de la pigmentación de la yema de huevo.

y se observó que presentan una coloración muy amarillo-rojizo; esto obedece a que en los concentrados comerciales se adicionan dichos carotenoides de forma sintética.

El color de la yema de huevo tiene un significado importante para el humano, considerando que la mayor intensidad lo da el contenido de carotenoides, que contribuyen al bienestar de la salud de quienes consumen huevos de gallina, entonces presenta mejora atributo si la pigmentación de la yema de este es producto de una alimentación de sostenéis natural u orgánica.

\section{f. Relación costo/beneficio}

Es necesario en toda unidad productiva hacer procesos de costos de producción para conocer el margen de utilidad que se obtiene. En la Tabla 1 se presentan los ingresos en dólares por venta de huevo en un ciclo de producción anual,y la venta de gallinas al terminar la producción de huevos. Los datos han sido proyectados por la producción de un ave.

Al determinar la relación costo/beneficio se puede afirmar que el mejor tratamiento fue el T1, considerando que éste tenía la dosis más baja de harina de hoja de Madre cacao, afirmando que por cada dólar de inversión se obtienen 20 centavos de dólar de utilidad. Esto podría resultar sustentable en las comunidades, partiendo de la autonomía en ingresos y la disponibilidad de alimento para el grupo familiar.

La producción de aves (gallinas) en las familias de las zonas rurales del país es un deno- 
Tabla 1. Proyecciones de rentabilidad económica según resultados del ensayo

\begin{tabular}{|l|c|c|c|c|}
\hline \multicolumn{1}{|c|}{ Tratamiento } & Costos de producción & $\begin{array}{c}\text { Ingre- } \\
\text { sos }\end{array}$ & Utilidad & B/C \\
\hline $\begin{array}{l}\text { T0: Harina de maíz } 49 \%+\text { harina de } \\
\text { sorgo } 49 \%+\text { minerales, vitaminas y } \\
\text { energía 2\%. }\end{array}$ & $\$ 30.37$ & $\$ 35.70$ & $\$ 5.33$ & $\$ 1.18$ \\
\hline $\begin{array}{l}\text { T1: Harina de maíz 46.5\%+ hari- } \\
\text { na de sorgo 46.5\% + harina Madre } \\
\text { cacao 5\% + minerales, vitaminas y } \\
\text { energía } \% .\end{array}$ & $\$ 28.16$ & $\$ 33.86$ & $\$ 5.70$ & $\$ 1.20$ \\
\hline $\begin{array}{l}\text { T2: Harina de maíz } 45.5 \%+\text { hari- } \\
\text { na de sorgo } 45.5 \%+\text { harina Madre } \\
\text { cacao } 7 \%+\text { minerales, vitaminas y } \\
\text { energía } \% .\end{array}$ & $\$ 27.87$ & $\$ 30.29$ & $\$ 2.42$ & $\$ 1.08$ \\
\hline $\begin{array}{l}\text { T3: Harina de maíz } 44 \%+\text { harina } \\
\text { de sorgo } 44 \%+\text { harina Madre ca- } \\
\text { cao } 10 \%+\text { minerales, vitaminas y } \\
\text { energía } \% .\end{array}$ & $\$ 29.87$ & $\$ 30.78$ & $\$ 0.91$ & $\$ 1.07$ \\
\hline
\end{tabular}

minador común sin embargo las familias lo hacen de forma cultural y no prestan atención a la rentabilidad que puede presentar un el tipo de ave que se tiene como un medio de vida en el hogar.

\section{Conclusiones}

Es importante destacar que el tratamiento con un 5\% de harina con hojas de Madre cacao y el del testigo con $0 \%$, resultaron estadísticamente iguales en producción de huevos. Este resultado se relaciona con el mayor consumo de las aves al concentrado que contenía bajo porcentaje de harina de madre cacao y no a los efectos del contenido nutricional de éste, ya que, en aquellos tratamientos con mayores porcentajes, las gallinas redujeron su consumo hasta 35.44 gramos de consumo diario. Según lo citado, el consumo promedio de concentrado en postura ronda los 3.75 onzas. Es importante que la adición de un suplemento no cambie el sabor del alimento al que están acostumbradas las aves.

Al hacer la comparación entre las razas se puede decir que Rodhe Island Red presenta mejor rusticidad y aceptación al consumo de concentrado artesanal, mayor producción de huevos; además, no presentó mortalidad. Mientras que la Plymouth Rock Barrada pre- 
sentó un $20 \%$ de mortalidad debido al poco consumo de alimento, aunque su comportamiento en peso fue mayor durante las diez semanas de evaluación. Se considera que esto se debe a sus características fenotípicas, ya que presentan mayor altura y cuerpo ancho.

El tratamiento con el 5\% de harina de hoja de Madre cacao presentó la mejor rentabilidad económica como resultado de una menor inversión al utilizar la hoja como recurso local.
En general se puede afirmar que la harina de hoja de Madre cacao influye en características cualitativas en la producción de huevo y carne; no tanto en variables cuantitativas como peso del ave, postura y peso del huevo, encontrando resultados iguales con el tratamiento que no se le adiciono esa harina.

\section{Referencias}

Aguilar, D. (2001). Evaluación de dos dietas en gallinas criollas y mejoradas en semi-confinamiento, sobre la postura en el municipio de Yuscarán, Honduras. Proyecto Especial Del Programa de Ingeniero .... Recuperado de https://scholar.google.com.sv/ scholar?hl=es\&q=Evaluacion $+\mathrm{de}+\mathrm{dos}+$ dietas $+\mathrm{en}+$ gallinas + criollas $+\mathrm{y}+$ mejoradas + en + semi+confinamiento $\% 2$ Csobre+la+postura+en+el+municipio+de+yuscaran $\%$ 2CHonduras\&btnG=\&lr=lang_es\#0

Cornejo, S., Hidalgo, H., Araya, J., y Pokniak, J. (2008). Suplementación de dietas de gallinas de postura comercial con aceites de pescado de diferentes grados de refinación: Efectos productivos en las aves y en la calidad organoléptica de los huevos. Archivos de Medicina Veterinaria, 40(1), 45-50. Recuperado de http://doi.org/10.4067/S0301732X2008000100006

Juárez-Caratachea, A., Gutiérrez-Vázquez, E., Segura-Correa, J., y Santos-Ricalde, R. (2010). Calidad del huevo de gallinas criollas criadas en traspatio en michoacan, mexico. Tropical and Subtropical Agroecosystems, 12(1), 109-115. Recuperado de http://www.redalyc.org/articulo.oa?id=93913074011

Marañón B., J. F. (2001). Evaluación de aves criollas y líneas mejoradas con dos tipos de alimentación sobre la postura en Zamorano, Honduras. Escuela Agrícola Panamericana, Zamorano. Recuperado de http://bdigital.zamorano.edu/handle/11036/1457 
Menchú, M., y Méndez, H. (2011). Análisis de la situación alimentaria en El Salvador. GuaTemala, Guatemala: Instituto de Recuperado de http://www.bvselsalvador.net/docs/ lildbi/495-an\%C3\%A1lisis-situacion-alimentaria.pdf

Sandoval, Romero y Eguaras, J. L. (1966) Efecto de los carotenoides sobre la pigmentación de la yema del huevo y la productividad de las aves. Escuela Nacional de Agricultura y Ganadería, Managua; Nicaragua

Salas, M., y Rodríguez, J. (2009). Producción de Huevo de Gallinas Rhode Island Rojas Bajo un Sistema Alternativo de Traspatio. Cadernos de Agroecología. Recuperado de http:// www.aba-agroecologia.org.br/revistas/index.php/cad/article/view/3630 